\title{
LANDAUER CONDUCTANCE OF GENERALIZED FIBONACCI-TYPE SEMICONDUCTOR SUPERLATTICES
}

\author{
W. Salejda, M. Kubisa, J. Misiewicz, K. Ryczko and M. Tyc \\ Institute of Physics, Wrocław University of Technology \\ Wybrzeze Wyspiańskiego 27, 50-370 Wrocław, Poland
}

\begin{abstract}
The quantum model of quasi-one-dimensional generalized Fibonacci semiconductor superlattice with the mass of charge carriers depending on the position in superlattice is formulated. The Landauer electrical conductance $\sigma_{\mathrm{L}}$ of generalized Fibonacci semiconductor superlattice is studied analytically and numerically. The dynamical maps allowing us to calculate $\sigma_{\mathrm{L}}$ of the studied systems are presented. It is shown that $\sigma_{\mathrm{L}}$ as a function of incident energy $E$ of charge carriers oscillates strongly and exhibits the resonant character. We have verified numerically that $\sigma_{\mathrm{L}}(E)$ reaches its local maximum for energies $E$ corresponding to energy eigenvalues of charges in superlattice.
\end{abstract}

PACS numbers: 73.40.-c, 73.20.Dx, 73.61.Ey

Recently in Refs. $[1,2]$ we have proposed and developed the dynamical maps approach to calculations of the Landauer conductance $\sigma_{\mathrm{L}}$ [3] of the quasi-one-dimensional generalized Fibonacci superlattices. Our results are an extension of the previously obtained maps [4] in a different context to the case of: (1) more generalized classes of aperiodic systems, (2) unimodular complex matrices. We found also a new algebraic proof of the trace maps derived in Ref. [4].

In this report we study the Landauer conductance (LC) of quasi-one-dimensional generalized Fibonacci semiconductor superlattice (GFSS) with nonconstant mass of charge carriers (CC).

Let us start with a short description of the construction of one-dimensional generalized Fibonacci lattice (GFL) [4]. Let $\mathcal{A}$ and $\mathcal{B}$ denote two segments, the lengths of which are $d_{a}$ and $d_{b}$, respectively. We construct GFL of type $(n, m)$ by the iterative process: $S_{1}=\mathcal{B}, S_{2}=\mathcal{A}, S_{l+1}=\left(S_{l}\right)^{n}\left(S_{l-1}\right)^{m}$, where $n$ and $m$ are natural numbers; the index $l$ defines the generation number of GFL, $\left(S_{l}\right)^{n}$ and $\left(S_{l-1}\right)^{m}$ mean $n$ and $m$ repetitions of $S_{l}$ and $S_{l-1}$, respectively.

We place the rectangular potential barrier with the height $U_{a}\left(U_{b}\right)$ and width $b_{a}\left(b_{b}\right)$ in the middle of segment $\mathcal{A}(\mathcal{B})$. We assume that the mass of charge carrier is equal to $\mu$ under the potential barrier and $m$ in the well region. Let us take the wave function in the form $\Psi(z)=A \exp (\mathrm{i} k z)+B \exp (-\mathrm{i} k z)$ at the left boundary 
of the segment (the left end of segment is placed at the beginning of $O Z$ axis and the centre of rectangular barrier has coordinate $d / 2$, in this way the barrier occupies the interval $[(d-b) / 2,(d+b) / 2]), \Psi(z)=A^{\prime} \exp (\beta z)+B^{\prime} \exp (-\beta z)$ in the potential barrier region and $\Psi(z)=C \exp [\mathrm{i} k(z-d)]+D \exp [-\mathrm{i} k(z-d)]$ at the right boundary. Applying the standard continuity conditions for the wave function $\Psi$ and its first derivative $(1 / m)(\mathrm{d} \Psi / \mathrm{d} z)$ we obtain the relation

$$
\left[\begin{array}{l}
C \\
D
\end{array}\right]=\left[\begin{array}{ll}
a_{11} & a_{12} \\
a_{11}^{*} & a_{11}^{*}
\end{array}\right]\left[\begin{array}{l}
A \\
B
\end{array}\right]=M\left[\begin{array}{l}
A \\
B
\end{array}\right],
$$

where the elements of the transfer matrix $M$ are given by

$$
\begin{aligned}
& a_{11}=\left[\cosh (\beta b)+\mathrm{i} \frac{k^{2} m^{2}-\beta^{2} \mu^{2}}{2 k m \beta \mu} \sinh (\beta b)\right] \exp [\mathrm{i} k(d-b)], \\
& a_{12}=-\mathrm{i} \frac{\beta^{2} m^{2}+k^{2} \mu^{2}}{2 k m \beta \mu} \sinh (\beta b)
\end{aligned}
$$

and $k^{2}=2 m E / \hbar^{2}, \beta^{2}=2 \mu(U-E) / \hbar^{2}, E$ denotes the energy of CC, $\mu(m)$ is the effective mass of CC in the barrier (well) region, $U=U_{a}\left(U_{b}\right), d=d_{a}\left(d_{b}\right)$, $b=b_{a}\left(b_{b}\right)$ for segment $\mathcal{A}(\mathcal{B})$. It is easy to verify that the matrix $M$ is unimodular, i.e., $\operatorname{det}(M)=1$.

The transfer matrix (TM) $\mathcal{M}_{1+1}$ for the whole Fibonacci structure of $(l+1)$-th generation is equal to the product of elementary transfer matrices $M_{j}$ (cf. Eq. (1)) corresponding to segments $\mathcal{A}$ and $\mathcal{B}$

$$
\begin{gathered}
\mathcal{M}_{l+1}=\left[\begin{array}{ll}
Y_{l+1}+\mathrm{i} Z_{l+1} & \left(\mathcal{M}_{l+1}\right)_{1,2} \\
\left(\mathcal{M}_{l+1}\right)_{1,2}^{*} & Y_{l+1}-\mathrm{i} Z_{l+1}
\end{array}\right] \\
=M_{N(l+1)} M_{N(l+1)-1} \cdot \ldots \cdot M_{2} M_{1},
\end{gathered}
$$

where $N(l+1)$ denotes the total number of segments in the studied structure, $Y_{l+1}=\operatorname{Re}\left(\mathcal{M}_{l+1}\right)_{1,1}, Z_{l+1}=\operatorname{Im}\left(\mathcal{M}_{l+1}\right)_{1,1}$. The Landauer conductance [3]

$$
\sigma_{\mathrm{L}}=\frac{e^{2}}{h\left|\left(\mathcal{M}_{l+1}\right)_{1,2}\right|^{2}}=\frac{e^{2}}{h\left[Y_{l+1}^{2}+Z_{l+1}^{2}-1\right]}
$$

can be calculated by means of product (2) or using the dynamical maps approach $[1,2]$

$$
\begin{aligned}
Y_{l+1} & =\mathcal{U}_{m-1}\left(Y_{l-1}\right) \mathcal{U}_{n-1}\left(Y_{l}\right) Y_{l}\left\{2 Y_{l-1}-\left[\frac{\mathcal{u}_{m-2}\left(Y_{l-1}\right)}{\mathcal{U}_{m-1}\left(Y_{l-1}\right)}+\frac{\mathcal{U}_{n-2}\left(Y_{l-1}\right)}{\mathcal{U}_{n-1}\left(Y_{l-1}\right)}\right]\right\} \\
& -\mathcal{U}_{m-1}\left(Y_{l-1}\right) \mathcal{U}_{n-1}\left(Y_{l}\right)\left[\frac{\mathcal{U}_{n-2}\left(Y_{l}\right)}{\mathcal{U}_{n-1}\left(Y_{l}\right)} Y_{l-1}+\frac{\mathcal{U}_{m-1}\left(Y_{l-2}\right)}{\mathcal{U}_{n-1}\left(Y_{l-1}\right)} Y_{l-2}\right] \\
& +\mathcal{U}_{m-1}\left(Y_{l-1}\right) \mathcal{U}_{n-1}\left(Y_{l}\right)\left[\frac{\mathcal{U}_{m-2}\left(Y_{l-2}\right)}{\mathcal{U}_{n-1}\left(Y_{l-1}\right)}+\frac{\mathcal{U}_{m-2}\left(Y_{l-1}\right)}{\mathcal{U}_{n-1}\left(Y_{l}\right)} \frac{\mathcal{U}_{n-2}\left(Y_{l}\right)}{\mathcal{U}_{m-1}\left(Y_{l-1}\right)}\right]
\end{aligned}
$$




$$
\begin{aligned}
Z_{l+1} & =\mathcal{U}_{n-1}\left(Y_{l}\right)\left[\frac{\mathcal{U}_{m-1}\left(Y_{l-1}\right) \mathcal{U}_{n-2}\left(Y_{l-1}\right)-\mathcal{U}_{m-2}\left(Y_{l-1}\right) \mathcal{U}_{n-1}\left(Y_{l-1}\right)}{\mathcal{U}_{n-1}\left(Y_{l-1}\right)}\right] Z_{l} \\
& +\mathcal{U}_{m-1}\left(Y_{l-1}\right) \mathcal{U}_{n}\left(Y_{l}\right) Z_{l-1} \\
& +\frac{\mathcal{U}_{m-1}\left(Y_{l-1}\right) \mathcal{U}_{m-1}\left(Y_{l-2}\right) \mathcal{U}_{n-1}\left(Y_{l}\right)}{\mathcal{U}_{n-1}\left(Y_{l-1}\right)}\left[\mathcal{U}_{n-1}^{2}\left(Y_{l-1}\right)+\mathcal{U}_{n-2}^{2}\left(Y_{l-1}\right)\right] Z_{l-2} \\
& -\mathcal{U}_{n-1}\left(Y_{l}\right) \mathcal{U}_{m-1}\left(Y_{l-2}\right) \mathcal{U}_{n-2}\left(Y_{l-1}\right)\left[\mathcal{U}_{m}\left(Y_{l-1}\right)+\mathcal{U}_{m-2}\left(Y_{l-1}\right)\right] Z_{l-2}
\end{aligned}
$$

where $\mathcal{U}_{n}(x)$ denotes the Chebyshev polynomial of the second order.

Let us specify the system under consideration. We put $U_{a}=0, U_{b}=U$, $b_{a}=0, b_{b}=d_{b}=b, d_{a}=a$. In this way we define the system containing two kinds of semiconductor layers distributed quasiperiodically. The potential wells correspond to the $\mathcal{A}$-type layers and the barriers to $\mathcal{B}$-type layers.

We have performed the numerical calculations for $n=m=1$. In Table we present a few lowest generations of simple Fibonacci semiconductor superlattices.

\section{TABLE}

A few initial Fibonacci-type semiconductor superlattices.

\begin{tabular}{c|l}
\hline \hline$l$ & \multicolumn{1}{|c}{$S_{l}$} \\
\hline 4 & $\mathcal{A B A}$ \\
5 & $\mathcal{A B A} \mathcal{A B}$ \\
6 & $\mathcal{A B A} \mathcal{B} \mathcal{B} \mathcal{A B A}$ \\
7 & $\mathcal{A B A} \mathcal{A} \mathcal{B} \mathcal{B} \mathcal{B} \mathcal{A} \mathcal{A B A} \mathcal{A} \mathcal{A B}$
\end{tabular}

Figure 1 presents the LC spectra calculated for the $\mathrm{GaAs} / \mathrm{Al}_{0.3} \mathrm{Ga}_{0.7} \mathrm{As}$ nanostructure when either the effective mass difference between these materials has been taken into account or not. We calculated also numerically eigenenergies being the solution of the eigenvalue problem $H \Psi_{j}=E_{j} \Psi_{j}$ for the structure under consideration. We imposed the boundary conditions $\Psi=0$ at the ends of the structure (which is equivalent to infinite potential barriers). The eigenvalues $E_{j}$ (for the case $m_{b} \neq m_{a}$ ) are plotted as the short vertical lines at the top of Fig. 1 .

The dimensionless conductance $T / R$ versus energy $E$ of $C C$ and the ratio $m_{b} / m_{a}$ is depicted in Fig. 2.

Figure 3 shows the Landauer conductance $T / R$ versus energy $E$ of $C C$ and the well width $a$. 


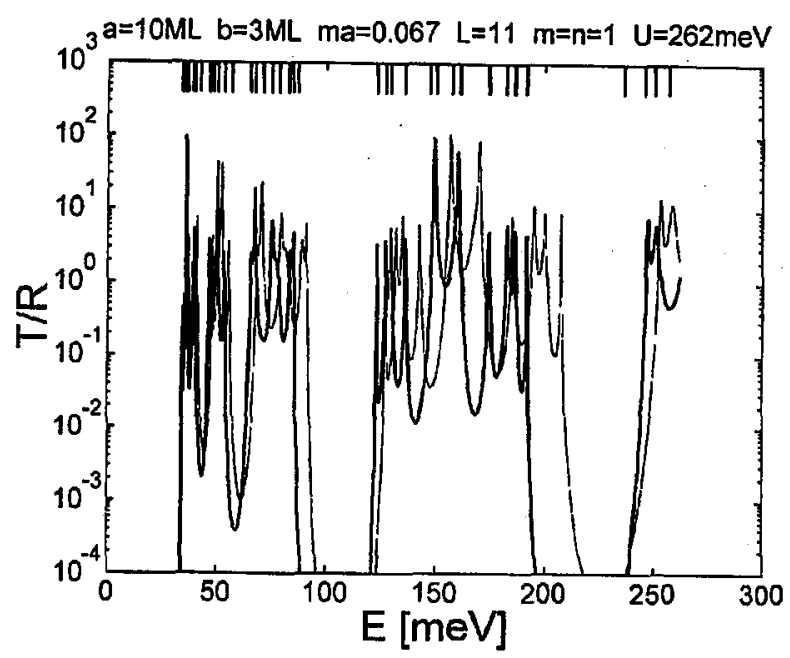

Fig. 1. The dimensionless Landauer conductivity $T / R$ versus electron energy $E$ at indicated values of model parameters. Thin line corresponds to the case $m_{b}=m_{a}=$ 0.067 , thick line - to the case $m_{b}=0.092, m_{a}=0.067$.

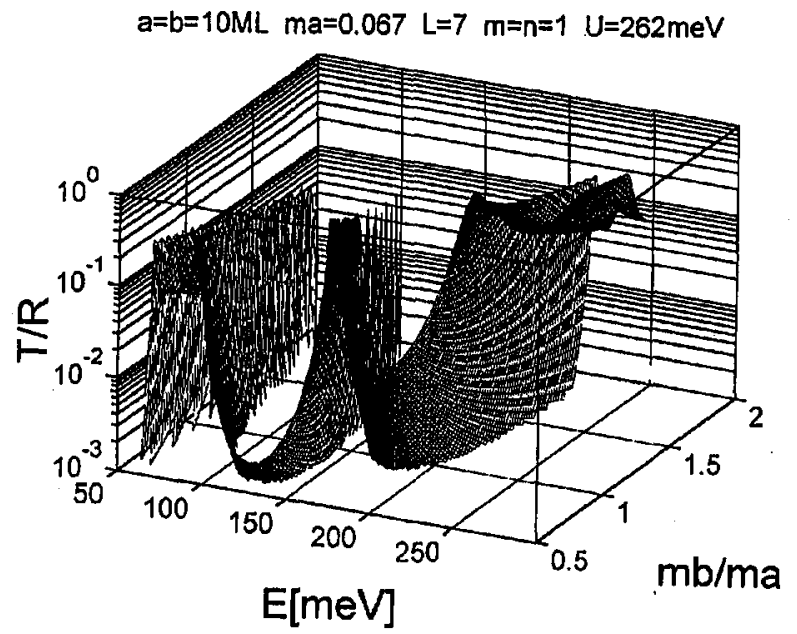

Fig. 2. The dependence of the dimensionless Landauer conductivity $T / R$ on electron energy $E$ and the ratio $m_{\mathrm{b}} / m_{\mathrm{a}}$ at the indicated values of model parameters.

We summarize the main results of the presented studies as follows:

1. The Landauer conductance as a function of incident energy of CC shows the resonant nature which is evident in all figures.

2. We observe correlation between maxima of $\sigma_{\mathrm{L}}(E)$ and eigenergies of CC in GFSS. Most of the computed energies correspond to resonance peaks in the LC spectrum. It can be verified that the other eigenstates are localized 


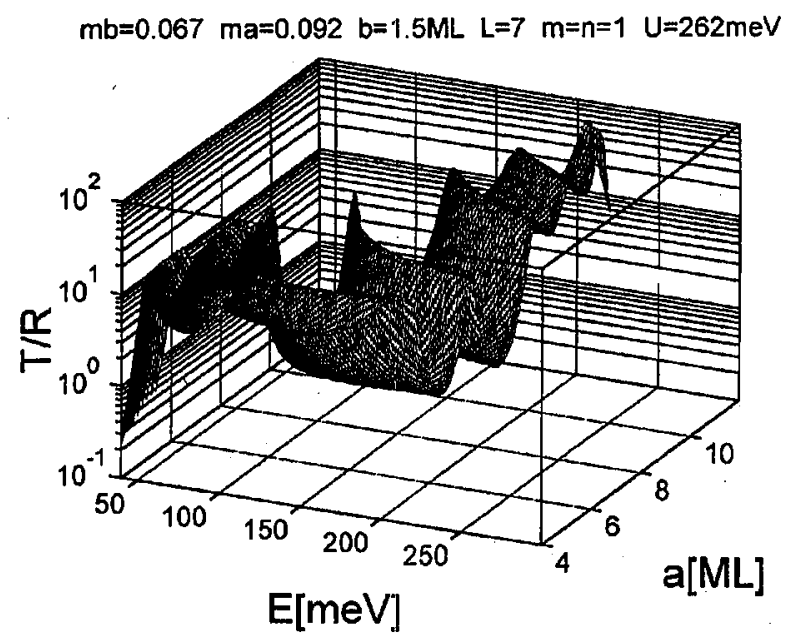

Fig. 3. The dependence of the dimensioness Landauer conductivity $T / R$ on electron energy $E$ and the well width $a$ at the indicated values of model parameters.

at the surface of the structure (they are the consequence of the boundary conditions).

3. Effective mass difference between well and barrier materials leads to a significant shift in the spectrum of LC (cf. Figs. 1 and 2).

\section{References}

[1] W. Salejda, Physica A 232, 769 (1996).

[2] W. Salejda, P. Szyszuk, Physica A 252, 547 (1998); W. Salejda, P. Szyszuk, Report Pre 174/96, Institute of Physics, Wrocław University of Technology, Wrocław 1996 (in Polish); P. Szyszuk, M.Sc. Thesis, Report SPR 327/97, Institute of Physics, Wrocław University of Technology, Wrocław 1997 (in Polish).

[3] R. Landauer, Z. Phys., Condens. Matter B 68, 217 (1987).

[4] G. Gumbs, M.K. Ali, Phys. Rev. Lett. 60, 1081 (1988); G. Gumbs, M.K. Ali, J. Phys., Math. Gen. A 21, L517 (1988); ibid. A 22, 951 (1988); M. Kolar, M.K. Ali, Phys. Rev. B 41, 7108 (1990); J.Q. You, J.R. Yan, T. Xie, X. Zeng, J.X. Zhong, J. Phys., Condens. Matter 3, 7255 (1991). 